\title{
Concurrent chemoradiotherapy with carboplatin followed by carboplatin and 5- fluorouracil in locally advanced nasopharyngeal carcinoma
}

\author{
Tanadech Dechaphunkul ${ }^{1 *}$, Kowit Pruegsanusak', Duangjai Sangthawan ${ }^{2}$ and Patrapim Sunpaweravong ${ }^{3}$
}

\begin{abstract}
Background: This study aimed to evaluate acute major toxicities, the response rate, 3-year overall survival and progression-free survival rate of locally advanced nasopharyngeal carcinoma patients on concurrent carboplatin chemoradiotherapy followed by carboplatin and 5-fluorouracil.

Methods: A prospective study of fifty patients diagnosed with locally advanced nasopharyngeal carcinoma received conventional radiation therapy with a total dose of 6600-7000 cGy in 6-7 weeks and concurrent chemotherapy of three cycles of carboplatin during radiotherapy, followed by adjuvant chemotherapy using carboplatin plus 5-fluorouracil for two cycles.

Results: Weight loss and mucositis were the two most common acute major grades 3-4 toxicities (42\%). Myelosuppression occurred subsequently, including leukopenia (30\%), neutropenia (20\%), anemia (12\%), and thrombocytopenia (6\%). Only 8\% of patients developed grades 3-4 nausea and vomiting. No patients had renal and electrolyte abnormalities. Regarding the response evaluation, 100\% of patients achieved an objective response rate of the primary tumor ( $92 \%$ complete response, and $8 \%$ partial response). Similarly, all patients also achieved an objective response rate of the neck node (64\% complete response and 36\% partial response). The 3-year overall survival rate and progression-free survival rate were $89.7 \%$ and $72.7 \%$, respectively.

Conclusions: Concurrent chemoradiotherapy with carboplatin followed by carboplatin and 5- fluorouracil could be considered as an alternative regimen for locally advanced nasopharyngeal carcinoma patients pertaining to a good overall response rate, 3-year overall survival and progression-free survival rate with good tolerability.
\end{abstract}

\section{Introduction}

Nasopharyngeal carcinoma is a common head and neck cancer in Southeast Asia. The incidence is high in the third to fifth decade of life with a male predominance [1]. In Songklanagarind Hospital, there were approximately 80-100 new cases per year. Most of the patients were diagnosed in the advanced stage of the disease (60-70\%).

According to the good response of radiotherapy (radiosensitive tumor) and anatomic restrictions, the standard treatment for nasopharyngeal carcinoma is definitive radiotherapy. However, $70 \%$ of patients presented with locally advanced stage cancer at the first

\footnotetext{
* Correspondence: dr.tanadech@gmail.com

'Department of Otorhinolaryngology Head and Neck Surgery, Faculty of

Medicine, Prince of Songkla University, Songkhla, Thailand

Full list of author information is available at the end of the article
}

time of diagnosis [2-5]. Moreover, it has been reported that nasopharyngeal carcinoma demonstrated the highest incidence of distant metastasis among all head and neck cancers [6-9]. The benefits of chemotherapy given concurrently with radiation are supported by the present data showing improvement in the effects of radiation through volume reduction, increased radiosensitizing effect, reduction of micro-metastasis, and overall survival improvement [10-12]. The first study was reported by the Head and Neck Intergroup 0099, using concurrent chemoradiotherapy with three cycles of cisplatin chemotherapy, followed by a 3-cycles combination of cisplatin and 5-fluorouracil chemotherapy. The results showed a significant benefit in both a progression-free survival and overall survival improvement [13]. Subsequently, there were several studies using the same combination of cisplatin and 5-fluorouracil chemotherapy as

\section{() Biomed Central}


a first-line treatment in patients with nasopharyngeal carcinoma, and switching from cisplatin to carboplatin whenever the patients developed renal or serious toxicities from cisplatin [1,13-18]. Additionally, there were some studies using carboplatin as the first-line drug and the results showed no significant differences in the overall response rate and overall survival rate when compared to cisplatin [19-21].

Regarding toxicity profiles, carboplatin has less adverse events in terms of nausea, vomiting, renal toxicity and ototoxicity, but it does have a more myelosuppressive effect when compared with cisplatin. There is also no need for fluid hydration for carboplatin administration, thus the total infusion time is significantly shorter resulting in being a good choice of chemotherapy particularly in an outpatient setting.

Based upon the previous data, the objective of this study is to evaluate the benefit of carboplatin chemotherapy on response rate, 3-year overall survival rate, 3-year progression-free survival rate, including toxicity as a first-line treatment in combination with radiotherapy in patients with locally advanced nasopharyngeal carcinoma.

\section{Materials and methods \\ 1) Patients}

Fifty patients diagnosed with locally advanced nasopharyngeal carcinoma who received concurrent chemoradiotherapy during May 2005 to December 2007 at Songklanagarind Hospital were evaluated. Eligible patients were those with histologically confirmed nasopharyngeal carcinoma with at least $\mathrm{T} 3$ or $\mathrm{N} 1 \geq 3 \mathrm{~cm}$ or $\geq \mathrm{N} 2$ as classified by the $6^{\text {th }}$ edition of the American Joint Committee on Cancer Staging System, and Eastern Cooperative Oncology Group (ECOG) performance status $0-2$. The exclusion criteria included patients who were more than 65 years of age, patients previously receiving treatment for a locally advanced disease, recurrent or metastatic disease, inadequate hematological profiles (absolute neutrophil count $<1500 / \mathrm{mm}^{3}$ or platelet count $<100,000 / \mathrm{mm}^{3}$ ), serum creatinine $>3 \mathrm{mg} /$ $\mathrm{dL}$, and poor compliance.

\section{2) Study Procedure}

All patients were evaluated by Head and Neck oncologist including complete history and physical examination, rigid nasopharyngoscopy, complete blood count, renal function, electrolyte and liver function tests. Radiological investigations included chest radiography and computed tomography or magnetic resonance imaging. An ultrasound of the hepatobiliary system was performed if there was a high level of serum alkaline phosphatase. This protocol was approved by the institutional ethics committees of Songklanagarind Hospital. All patients provided written informed consent.
Conventional radiation therapy was used in all patients by $200 \mathrm{cGy}$ per fraction with five daily fractions per week to a total dose of 6600-7000 cGy. A concurrent chemotherapy regimen was given by medical oncologist using carboplatin (AUC 6) intravenously every 3 weeks for 3 cycles during radiotherapy, followed by 2 cycles more of chemotherapy using carboplatin (AUC 5) plus 5 - fluorouracil (1,000 $\mathrm{mg}$ per $\mathrm{m}^{2}$ per day by 4 days infusion) every 3 weeks. The treatment protocol was shown in Figure 1. Dose modifications were performed according to patient's toxicity grading. Subsequent doses of chemotherapy were reduced by $20 \%$ if the patients developed grade 3 or 4 adverse events.

Patient's toxicities were graded by the Radiation Therapy Oncology Group toxicity criteria during treatment. The assessment of the tumor response was performed 3 weeks after the last cycle of adjuvant chemotherapy was completed including a complete physical examination, rigid nasopharyngoscopy and computed tomography or magnetic resonance imaging according to the Response Evaluation Criteria In Solid Tumor (RECIST) criteria. Patients were followed up every 2 months in the first year, every 3 months in the second year, every 4 months in the third year and then every 6-12 months until death. The treatment of a residual tumor was performed based upon the guidelines of our hospital.

\section{Statistical Analysis}

In the statistical analysis, the epidemiology, clinical characteristics, and outcome data were assessed using the Fisher's exact test and the estimated survival probability was assessed with the Kaplan-Meier method by the $\mathrm{R}$ software package Epicalc.

\section{Results}

Fifty patients diagnosed with locally advanced nasopharyngeal carcinoma were included in this study. All patients received concurrent chemoradiotherapy with a carboplatin agent.

The mean age of the patients was 44 years with a range of 22 to 62 years. The majority of patients were male (68\%), AJCC stage III and IV (92\%), and WHO histopathology type 1 (52\%). Regarding cancer risk factors, only $16 \%$ of patients had a significant family history of cancer, however $50 \%$ of patients were active smokers and $44 \%$ were alcohol consumers. Most patients had an excellent ECOG performance status before treatment. Ninety-eight percent of patients could receive a completion of 5 planned cycles of chemotherapy. (Table 1)

The most common symptom was a neck mass (82\%). The other subsequent complaints included aural fullness (62\%), nasal obstruction (42\%), epistaxis (16\%) and bloody sputum (14\%). 


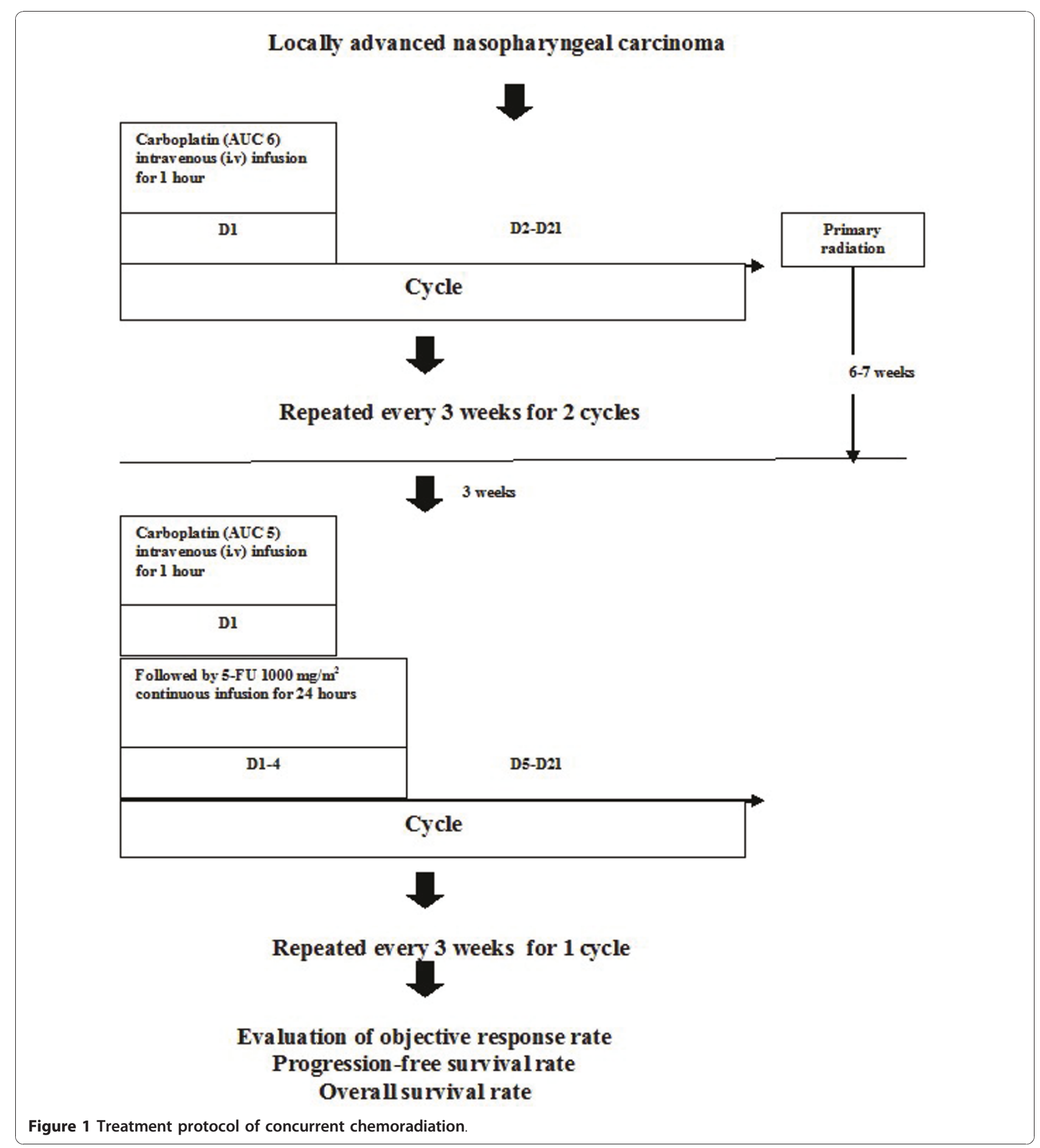

Weight loss (42\%) and mucositis (42\%) were the most two common moderate to severe acute toxicities that was found in this protocol. Severity of mucositis was demonstrated in Figure 2 and 3. Myelosuppression occurred subsequently, including leukopenia (30\%), neutropenia (20\%), anemia (12\%) and thrombocytopenia $(6 \%)$. Only $8 \%$ of patients had moderate to severe nausea and vomiting and no patients had renal or electrolyte abnormalities from this treatment. (Table 2)

Regarding the response evaluation, both the primary site and neck node had achieved an objective response rate of $100 \%$. The primary site showed a $92 \%$ complete response and partial response was $8 \%$. Sixty-four percent of neck node sites showed a complete response and $36 \%$ 


\section{Table 1 Patient Characteristics}

\begin{tabular}{|c|c|}
\hline Characteristics & Number of patients (\%) \\
\hline Age & $44(22-62)$ \\
\hline \multicolumn{2}{|l|}{ Sex } \\
\hline - Male & $34(68 \%)$ \\
\hline - Female & $16(32 \%)$ \\
\hline \multicolumn{2}{|c|}{ Family history of cancer } \\
\hline - Yes & $8(16 \%)$ \\
\hline$-\mathrm{No}$ & $42(84 \%)$ \\
\hline \multicolumn{2}{|l|}{ Active smoking } \\
\hline - Yes & $25(50 \%)$ \\
\hline - No & $25(50 \%)$ \\
\hline \multicolumn{2}{|c|}{ Alcohol consumer } \\
\hline - Yes & $22(44 \%)$ \\
\hline - No & $28(56 \%)$ \\
\hline \multicolumn{2}{|l|}{ Staging } \\
\hline - Stage IIb & $4(8)$ \\
\hline - Stage III & $18(36)$ \\
\hline - Stage IVa & $19(38)$ \\
\hline - Stage IVb & $9(18)$ \\
\hline \multicolumn{2}{|l|}{ T stage } \\
\hline$-\mathrm{T1}$ & $9(18)$ \\
\hline$-\mathrm{T} 2 \mathrm{a}$ & $3(6)$ \\
\hline$-\mathrm{T} 2 \mathrm{~b}$ & $12(24)$ \\
\hline$-\mathrm{T3}$ & $7(14)$ \\
\hline$-\mathrm{T} 4$ & $19(38)$ \\
\hline \multicolumn{2}{|l|}{$\mathrm{N}$ stage } \\
\hline - NO & $2(4)$ \\
\hline$-N 1$ & $9(18)$ \\
\hline$-N 2$ & $30(60)$ \\
\hline - N3a & $6(12)$ \\
\hline$-N 3 b$ & $3(6)$ \\
\hline \multicolumn{2}{|c|}{ Histopathology (WHO) } \\
\hline - Type 1 & $26(52)$ \\
\hline - Type 2 & $1(2)$ \\
\hline - Type 3 & $23(46)$ \\
\hline \multicolumn{2}{|c|}{ ECOG performance status } \\
\hline-0 & $45(90)$ \\
\hline-1 & $2(4)$ \\
\hline-2 & $3(6)$ \\
\hline \multicolumn{2}{|c|}{ Courses of chemotherapy } \\
\hline - 4 courses & $1(2)$ \\
\hline-5 courses & $49(98)$ \\
\hline
\end{tabular}

had a partial response. No patients had a stable and progressive disease. (Table 3)

The median follow-up time was 37.3 months (range 359 months). (The 3-year overall survival rate and progression-free survival rate were $89.7 \%$ and $72.7 \%$, respectively. (Figure 4, 5)

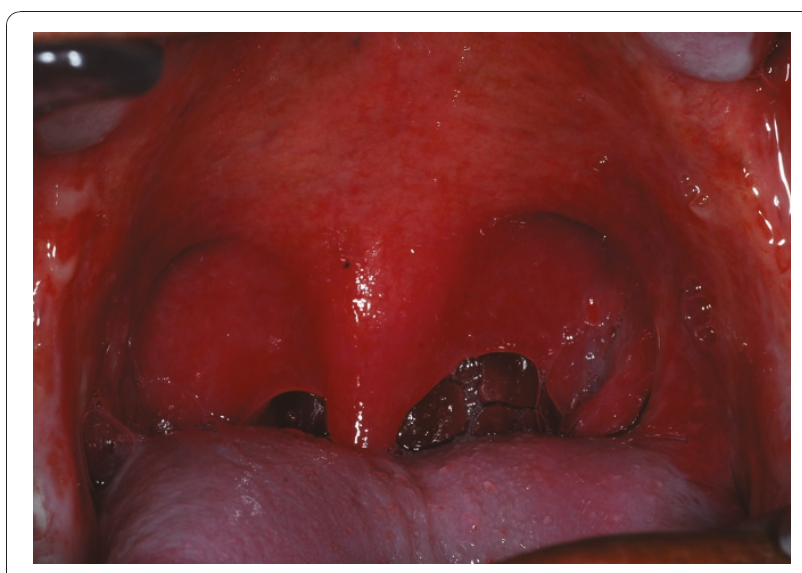

Figure 2 Grade 1 mucositis, erythematous mucosa.

\section{Discussion}

Nasopharyngeal carcinoma is a common head and neck cancer in Southeast Asia and more than $70 \%$ of patients presented with locally advanced stage cancer. According to the complexity of nasopharyngeal cancer treatment as well as the potential adverse events, a multidisciplinary approach is essentially encouraged. This requires the involvement of a head and neck oncologist, radiation oncologist and medical oncologist to achieve the best treatment outcome for individual patients. In our practice, the decision pathway is as shown in Figure 6.

Nowadays, concurrent chemoradiotherapy is the standard treatment for locally advanced nasopharyngeal carcinoma. Cisplatin chemotherapy was widely used as a first-line agent in combination with radiotherapy for nasopharyngeal carcinoma, whereas carboplatin would be considered as the option in case of intolerable side effects from cisplatin, especially ototoxicity and nephrotoxicity. Therefore, the objective of this study is to evaluate the benefit and safety profiles of carboplatin

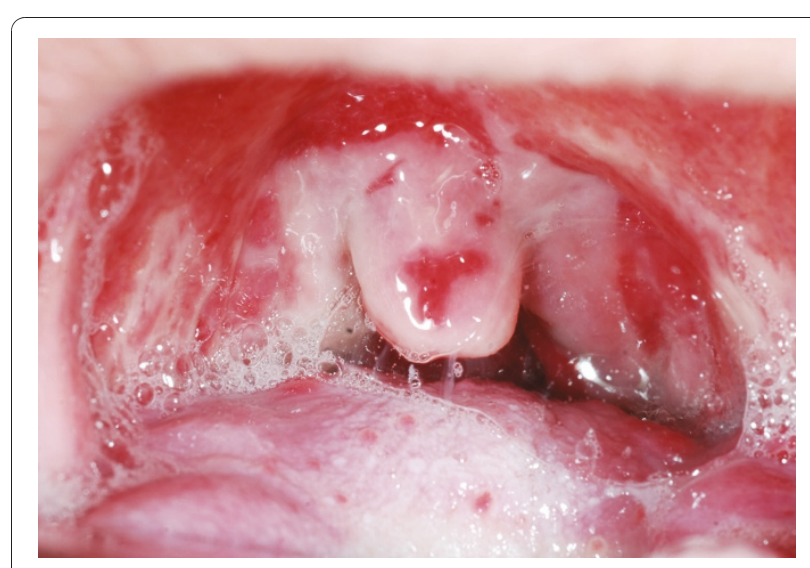

Figure 3 Grade 3 mucositis, confluent pseudomembanous mucosa. 
Table 2 Acute major toxicity profiles $(\mathbf{N}=\mathbf{5 0})$

\begin{tabular}{lcc}
\hline \multirow{2}{*}{ Toxicity } & \multicolumn{2}{c}{ Grading (No. (\%)) } \\
\cline { 2 - 3 } & Grade 1-2 & Grade 3-4 \\
\cline { 2 - 3 } (Mild) & (Moderate to severe) \\
\hline Hematologic toxicity & $44(88)$ & $6(12)$ \\
\hline Themia & $30(60)$ & $3(6)$ \\
\hline Leukopenia & $31(62)$ & $15(30)$ \\
\hline Neutropenia & $30(60)$ & $10(20)$ \\
\hline Non-hematologic toxicity & & $4(8)$ \\
\hline Nausea & $35(70)$ & $4(8)$ \\
\hline Vomiting & $31(62)$ & $21(42)$ \\
\hline Mucositis & $29(58)$ & $8(16)$ \\
\hline Fatigue & $41(82)$ & $21(42)$ \\
\hline Weight loss & $29(58)$ & $4(8)$ \\
\hline Skin & $46(92)$ & $0(0)$ \\
\hline Hypokalaemia & $29(58)$ & $0(0)$ \\
\hline Hyponatremia & $15(30)$ & $0(0)$ \\
\hline Renal toxicity & $2(4)$ &
\end{tabular}

chemotherapy in combination with radiotherapy as a first-line treatment in these patients.

Our study strongly supported that carboplatin chemotherapy can be considered as a first-line alternative treatment for locally advanced nasopharyngeal carcinoma patients or switching treatment in patients who experienced the intolerable adverse events from cisplatin chemotherapy. There are many advantages in using carboplatin as a treatment in patients with locally advanced nasopharyngeal carcinoma. Firstly, our study showed comparable acute major toxicity between carboplatin and cisplatin chemotherapy, however there were less nausea, vomiting and renal impairment in favoring carboplatin chemotherapy. Mucositis was the most common acute major toxicity found in our study with $42 \%$ serious adverse events. Previous reports with cisplatin regimen showed mucositis was the most common acute major toxicity (61\%), subsequently to leukopenia (32\%), anemia (19\%), thrombocytopenia (2\%), vomiting (18\%), renal impairment (2\%) and hyponatremia (2\%) [22]. However, thrombocytopenia was found to be more

Table 3 Response evaluation after complete treatment

\begin{tabular}{lc}
\hline Response evaluation & Number of patients (\%) \\
\hline Primary & \\
\hline - Complete response & $47(94)$ \\
\hline - Partial response & $3(6)$ \\
\hline Neck node & \\
\hline - Complete response & $32(64)$ \\
\hline - Partial response & $18(36)$ \\
\hline
\end{tabular}

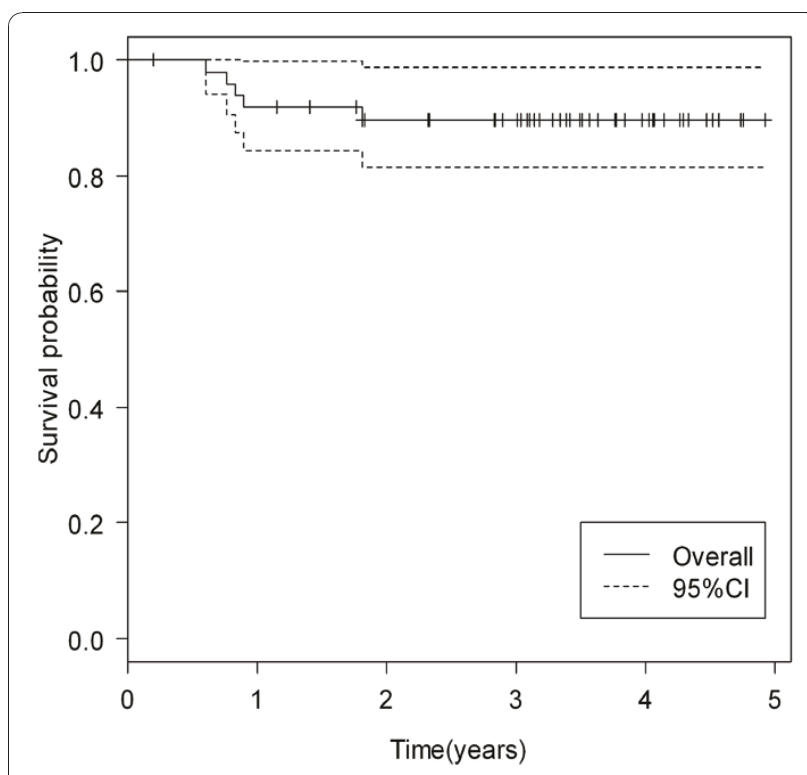

Figure 4 3-year overall survival rate of locally advanced nasopharyngeal carcinoma patients.

common in our study (6\%). Thus, oncologists should be aware of this common adverse event and symptomatic treatment should be adequately offered to patients, which may eventually have an impact on the patients' compliance to therapy and their quality of life.

Secondly, our study with a carboplatin regimen showed good patient's compliance and tolerability. 98\% of patients could receive a completion of 5 planned

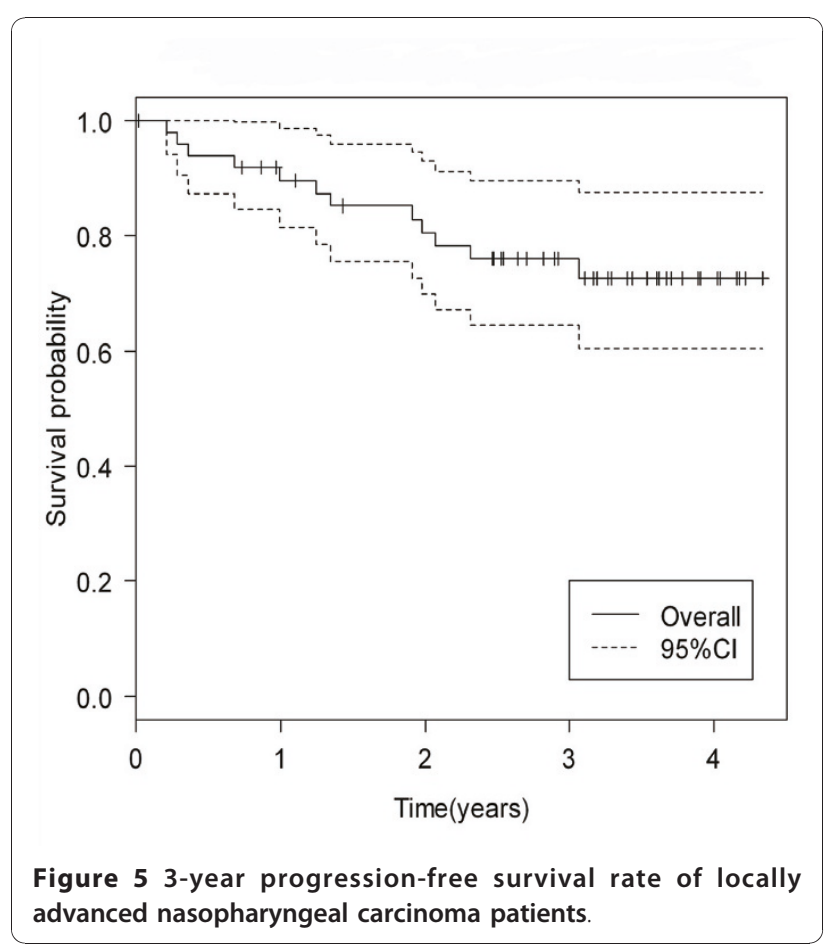




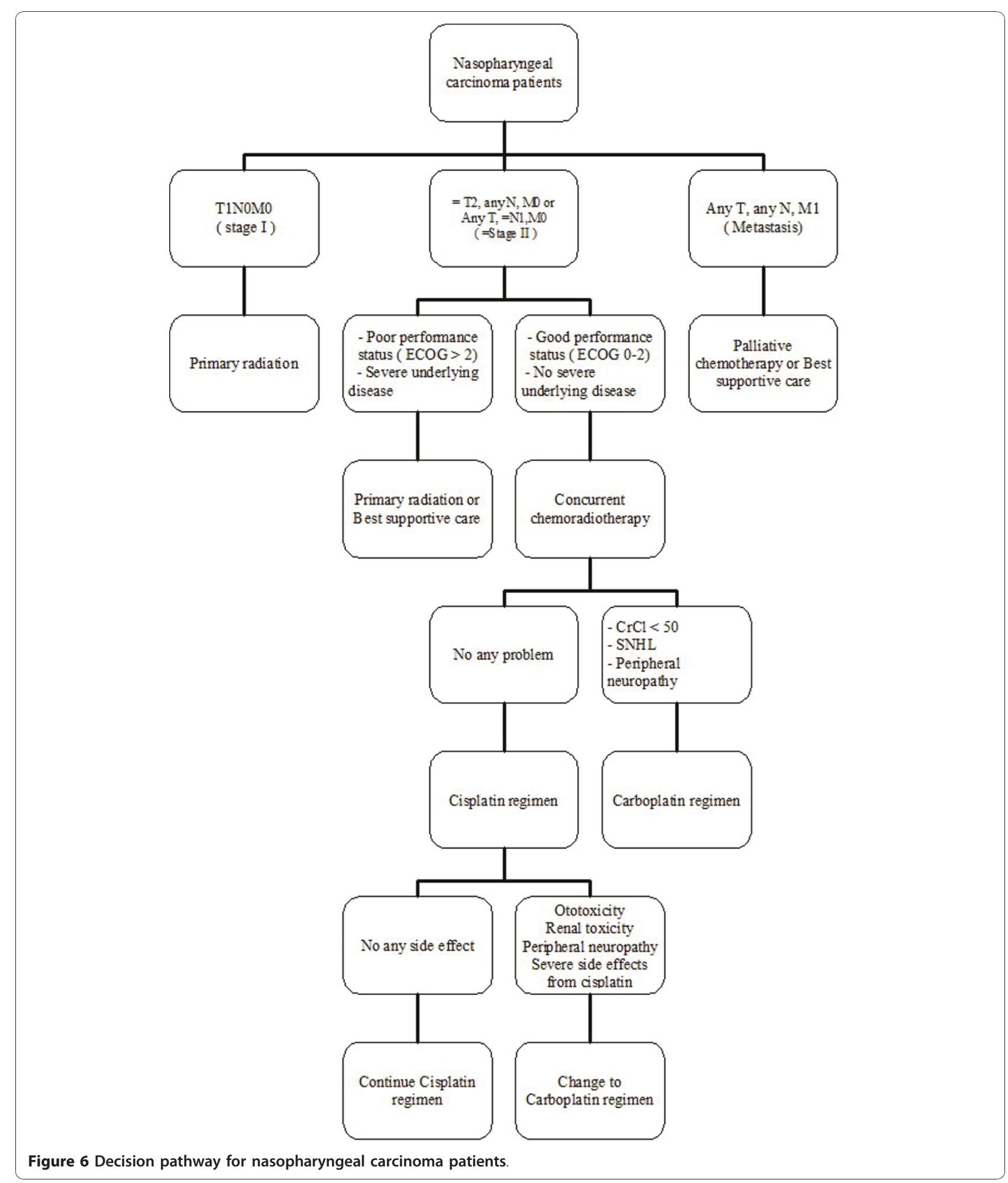

cycles of carboplatin chemotherapy. This is similar to another report which showed that the carboplatin regimen had a better tolerability than the cisplatin regimen [21]. Moreover, the carboplatin regimen took less time for chemotherapy infusion as there was no pre and post hydration. As a result, carboplatin could be considered the better option for outpatient cases.

Subsequently, carboplatin chemotherapy also demonstrated a comparable response rate to cisplatin. Data from previous literature on concurrent chemoradiotherapy with 
cisplatin regimen showed there was a wide range of responses between $49 \%$ to $98 \%$ [13,17,23-25]. In our study, the objective response rate of both the primary site and neck node showed 100\%, however the complete response rate of the primary site was $92 \%$ and neck node site with a complete response showed only $64 \%$. As such, carboplatin could be the acceptable alternative agent used for the treatment of nasopharyngeal carcinoma.

Finally, our data indicated a good overall survival and progression-free survival rate from concurrent chemotherapy with carboplatin chemotherapy with no substantially difference from cisplatin. The three-year overall survival rate and progression-free survival rate in nasopharyngeal carcinoma patients who received concurrent chemoradiotherapy with cisplatin were 71-89\%, and $54-88 \%$, respectively $[10,17,21]$. These were similar to our results showing $89.7 \%$ of a 3 -year overall survival rate and $72.7 \%$ of a 3 -year progression-free survival rate. Similarly, the recent data supported the benefit of carboplatin chemotherapy revealing no difference in the overall survival rate and progression-free survival rate between cisplatin and carbplatin chemotherapy given concurrently with radiation [21].

On the other hand, the nasopharyngeal carcinoma patients who had a residual neck node after treatment had to have performed salvage neck dissection according to the guidelines. However, there were some patients who needed to have their neck nodes observed and it was found that their neck nodes had subsided when follow up imaging was done 3-6 months later. Therefore, it may be that tumor evaluation should be extended to more than 3 weeks after the complete adjuvant chemotherapy for the actually tumor response or more accurate imaging such as PET-CT should be considered to decrease the likelihood of an unnecessary neck dissection.

In summary, our data strongly supported the benefit of concurrent carboplatin chemotherapy with radiotherapy for locally advanced nasopharyngeal carcinoma patients pertaining to a good overall response rate, 3year over all survival and progression-free survival rate with good tolerability. It can be considered as an alternative first line optional therapeutic regimen for this patient group.

\section{Acknowledgements \\ This study was supported by grants from Faculty of Medicine, Prince of Songkla University}

\section{Author details}

${ }^{1}$ Department of Otorhinolaryngology Head and Neck Surgery, Faculty of Medicine, Prince of Songkla University, Songkhla, Thailand. 'Division of Radiation Oncology, Department of Radiology, Faculty of Medicine, Prince of Songkla University, Songkhla, Thailand. ${ }^{3}$ Division of Medical Oncology, Department of Internal Medicine, Faculty of Medicine, Prince of Songkla University, Songkhla, Thailand.

\section{Authors' contributions}

TD carried out all data base collection, participated in study design, performed the treatment, did follow-up and statistical analysis. KP participated in its design, performed the treatment, and did follow-up. DS and PS performed the treatment, and did follow-up. All authors read and approved the final manuscript.

\section{Competing interests}

The authors declare that they have no competing interests.

Received: 9 May 2011 Accepted: 5 June 2011 Published: 5 June 2011

\section{References}

1. Chan AT, Teo PM, Leung TW, Johnson PJ: The Role of Chemotherapy in the Management of Nasopharyngeal Carcinoma. Cancer 1998, 82:1003-1012.

2. Skinner DW, Van Hasselt CA: Nasopharyngeal carcinoma: methods of presentation. Ear Nose Throat J 1990, 69:237-240.

3. Mackie AM, Epstein JB, Wu JS, Stevenson-Moore P: Nasopharyngeal carcinoma: The role of the dentistin assessment, early diagnosis and care before and after cancer therapy. Oral Onco 2000, 36:397-403.

4. Chen MS, Lin FJ, Tang SG, Leung WM, Leung W: Clinical significance of cranial nerve deficit in the therapy of nasopharyngeal carcinoma. Brit Radiol 1989, 62:739-743.

5. Epstein JB, Jones CK: Presenting sign and symptoms of nasopharyngeal carcinoma. Oral surg Oral med Oral pathol 1993, 75:32-36.

6. Chen WZ, Zhou DL, Luo KS: Long-term observation after radiotherapy for nasopharyngeal carcinoma. Int J Radiat Oncol Biol Phys 1989, 16:311-314.

7. Fandi A, Altun M, Azli N, Armand JP, Cvitkovic E: Nasopharyngeal carcinoma:epidemiology, staging, and treatment. Semin Oncol 1994, 21:382-397.

8. Vikram B, Mishra UB, Strong EW, Manolatos S: Patterns of failure in carcinoma of the nasopharynx: failure at distant sites. Head Neck Surg 1986, 8:276-279.

9. Zhang EP, Lian PG, Cai KL, Chen YF, Cai MD, Zheng XF, Guang XX: Radiation therapy of nasopharyngeal carcinoma: prognostic factors based on a 10-year follow-up of 1302 patients. Int J Radiat Oncol Biol Phys 1989, 16:301-305.

10. Afqir S, Ismaili N, Errihini H: Concurrent chemoradiotherapy in the management of advanced nasopharyngeal carcinoma: Current status. J Cancer Res Ther 2009, 5:3-7.

11. Baujat B, Audry H, Bourhis J, Chan AT, Onat H, Chua DT, Kwong DL, AlSarraf M, Leung SF, Thephamongkol K, Pignon JP, MAC-NPC Collaborative Group: Chemotherapy as an adjunct to radiotherapy in locally advanced nasopharyngeal carcinoma. Cochrane Database Syst Rev 2006, 18 : CD004329.

12. Baujat B, Audry H, Bourhis J, Chan AT, Onat H, Chua DT, Kwong DL, AlSarraf M, Chi KH, Hareyama M, Leung SF, Thephamongkol K, Pignon JP, MAC-NPC Collaborative Group: Chemotherapy in locally advanced nasopharyngeal carcinoma: an individual patient data meta-analysis of eight randomized trials and 1753 patients. Int J Radiat Oncol Biol Phys 2006, 64:47-56.

13. Al-Sarraf M, LeBlanc M, Giri PG, Fu KK, Cooper J, Vuong T, Forastiere AA, Adam G, Sakr WA, Schuller DE, Ensley JF: Chemoradiotherapy versus radiotherapy in patients with advanced nasopharyngeal cancer: phase III randomized Intergroup study 0099. J Clin Oncol 1998, 16:1310-1307.

14. Serin M, Erkal HS, Erkal Cakmak A: Radiation Therapy and Concurrent Cisplatin in Management of Locoregionally Advanced Nasopharyngeal Carcinomas. Acta Oncologica 1999, 38:1031-1035.

15. Chua DT, Sham JS, Au GK, Choy D: Concomitant chemoirradiation for stage III-IV nasophayngeal carcinoma in Chinese patients: results of a matched cohort analysis. Int J Radiat Oncol Biol Phys 2002, 53:334-343.

16. Daniilidis J, Fountzilas G: Combined radiochemotherapy in locally advanced nasopharyngeal carcinoma. HNO 2001, 49:732-738.

17. Wee J, Tan EH, Tai BC, Wong HB, Leong SS, Tan T, Chua ET, Yang E, Lee KM, Fong KW, Tan HS, Lee KS, Loong S, Sethi V, Chua EJ, Machin D: Randomized trial of radiotherapy versus concurrent chemoradiotherapy followed by adjuvant chemotherapy in patients with American Joint Committee on Cancer/International Union against cancer stage III and IV nasopharyngeal cancer of the endemic variety. J Clin Oncol 2005, 23:6730-6738. 
18. Maoleekoonpairoj S, Phromratanapongsa P, Puttanuparp S: Phase II study: Concurrent chemo-Radiotherapy in Advanced nasopharyngeal Carcinoma. J Med Assoc Thai 1997, 80:778-784.

19. Paliament M, Jha N, Rapp E, Smith C, MacKinnon J, Nabholtz JM, Hanson J, Reiman T, Mackey J: Concurrent weekly carboplatin and radiotherapy for nasopharyngeal carcinoma: report of a joint phase II study. Radiother oncol 2001, 58:131-136.

20. Okita J, Hatta C, Terada T, Saeki N, Ogasawara H, Kakibuchi M, Kamikonya N, Sakagami M: Concurrent chemo-radiotherapy for nasopharyngeal carcinoma. Auris Nasus Larynx 2004, 31:43-47.

21. Chitapanarux I, Lorvidhaya V, Kamnerdsupaphon P, Sumitsawan Y, Tharavichitkul E, Sukthomya V, Ford J: Chemoradiation comparing cisplatin versus carboplatin in locally advanced nasopharyngeal cancer: Randomised, non-inferiority, open trial. Eur J Cancer 2007, 43:1399-1406.

22. Lee AW, Tung SY, Chua DT, Ngan RK, Chapell R, Tung R, Siu L, Nq WT, Sze WK, Au GK, Law SC, O'Sullivan B, Yau TK, Leung TW, Au JS, Sze WM, Choi CW, Fung KK, Lau JT, Lau WH: Randomized Trial of Radiotherapy Plus Concurrent-Adjuvant Chemotherapy vs Radiotherapy Alone for Regionally Advanced Nasopharyngeal Carcinoma. J Natl Cancer Inst 2010, 102:1-11.

23. Lin JC, Jan JS, Hsu CY, Liang WM, Jiang RS, Wang WY: Phase III study of concurrent chemoradiotherapy versus radiotherapy alone for advanced nasopharyn-geal carcinoma: positive effect on overall and progressionfree survival. J Clin Oncol 2003, 21:631-637.

24. Chan AT, Teo PM, Ngan RK, Leung TW, Lau WH, Zee B, Leung SF, Cheung FY, Yeo W, Yiu HH, Yu KH, Chiu KW, Chan DT, Mok T, Yuen KT, Mo F, Lai M, Kwan WH, Choi P, Johnson PJ: Concurrent chemotherapyradiotherapy compared with radiotherapy alone in locoregional advanced nasopharyngeal carcinoma: progression-free survival analysis of a phase III randomized trial. J Clin Oncol 2002, 20:2038-2044.

25. Krstevska V, Stojkovski I, Klisarovska V: Concurrent chemoradiotherapy in locally and/or regionally advanced nasopharyngeal carcinoma. Prilozi 2008, 29:295-307.

doi:10.1186/1758-3284-3-30

Cite this article as: Dechaphunkul et al:: Concurrent chemoradiotherapy with carboplatin followed by carboplatin and 5- fluorouracil in locally advanced nasopharyngeal carcinoma. Head \& Neck Oncology 2011 3:30.

\section{Submit your next manuscript to BioMed Central and take full advantage of:}

- Convenient online submission

- Thorough peer review

- No space constraints or color figure charges

- Immediate publication on acceptance

- Inclusion in PubMed, CAS, Scopus and Google Scholar

- Research which is freely available for redistribution

Submit your manuscript at www.biomedcentral.com/submit
Biomed Central 